\title{
Shadow Elimination of Moving Targets Base on a Novel Two-Step Algorithm
}

\author{
Liu Ying*
}

\author{
Chongqing Jiaotong University, Chongqing, 400074, P.R. China
}

\begin{abstract}
This paper presents a two-step method for eliminating the shadows of moving targets. As the first step, we improve the Gaussian mixture model for obtaining a real-time background and a moving foreground; secondly, we figure out the intersection of background difference and moving foreground for eliminating the concomitant shadow [1-5]. Experiment results indicate that our method has a preferable performance on eliminating the shadow in its entirety.
\end{abstract}

Keywords: Elimination, gaussian mixture model, moving targets, shadow.

\section{INTRODUCTION}

In nature, a moving target carries its own shadow owing to the illumination of light (either artificial or from the sun) in circumstances. The shadow size is decided on the relative position of light source and the target. As the shadow area is large at a certain time, it will directly affect the extraction accuracy of a moving target and cause distortion.

The Gaussian mixture model proposed by Grimson and Stauffer is one of the most widely used tools for moving object extraction, but not so effective in processing the shadow of a moving target [6-8].

How the Gaussian mixture model for target extraction is capable of dealing with shadow is given in Table $\mathbf{1}$.

As we can see, obvious shadows in the 340th and 398th frames are detrimental for the extraction of moving objects [4].

\section{IMPROVED MODEL FOR SHADOW REDUCING}

Firstly, we attempt to obtain a real-time background and a moving foreground by improving the Gaussian mixture model, and then work out the intersection of background difference and moving foreground so as to reduce the influence of the target shadow on the result of test.

The specific establishment of our model and updated algorithm can be described as follows:

For image $\mathrm{I}$, we represent $\mathrm{x}$ as pixel and $\mathrm{t}$ as time, we simulate the process of each pixel $\mathrm{x}$ using $\mathrm{K}$ models $\mathrm{M}$.

$\left\{M_{1}, M_{2} \ldots M_{\mathrm{K}}\right\}(3 \leq K \leq 5)$

For each model, assuming its mean value is $\mu$, its weight is $\omega$, its survival time is $l$, and the frequency of occurrence is $f$.
To initialize the Gauss model, we use the first image frame to build a model for each pixel. In the first place, parameter $\mu$ is set as the value of pixel, which means color or gray level, the weight value $\omega$ is 1 , survival time $l$ is 175 , and the frequency of occurrence $f$ is 0 .

Then we use the new pixel values $P_{t}(x, y)$ to match the $\mathrm{K}^{\text {th }}$ Gauss distribution. If formula 1 is satisfied.

$\left|P_{t}(x, y)-\mu_{t, i}(x, y)\right| \leq T h_{t, i}$

We reckon $P_{t}(x, y)$ as matching ith Gauss distribution. Herein, $T h_{t, i}$ means time $t$, and matching threshold of the ith Gauss model is determined by formula 2 .

$T h_{t}=\left(\max \left(P_{t}(x, y)\right)-\min \left(P_{t}(x, y)\right) / D I V\right.$

There in $D I V$ is the proportional coefficient. The greater the $D I V$, the lower the threshold, and the higher the resolution of model. Matching threshold of the model is proportional to the color change of current frame. $\max \left(P_{t}(x\right.$, $y)$ is the maximum value of pixel in the frame of the time t, while $\min \left(P_{t}(x, y)\right.$ is the minimum. Because of influence from real environment, noise is inevitable, and brightness and color of the video frames are also in constant change. If a fixed threshold is employed in the method, the system resolution will be reduced when there are obvious changes in the image. Associating the threshold value with the current video frame $[8,9]$, we can ensure that the resolution of the system is also maintained at the same level. Therefore, setting of the matching threshold is reasonable.

In the initialization phase of the Gauss model, if the pixel $P_{t}(x, y)$ of the time $\mathrm{t}$ matches the ith model, then we update the model parameter as follows:

$$
\begin{aligned}
& \omega_{t+1, i}(x, y)=\omega_{t, i}(x, y)+1 \\
& \mu_{t+1, i}(x, y)=\mu_{t, i}(x, y)+\operatorname{sgn}\left(P_{t}(x, y)-\mu_{t, i}(x, y)\right)
\end{aligned}
$$


Table 1. Results of Gaussian mixture model for real video processing (1-1).

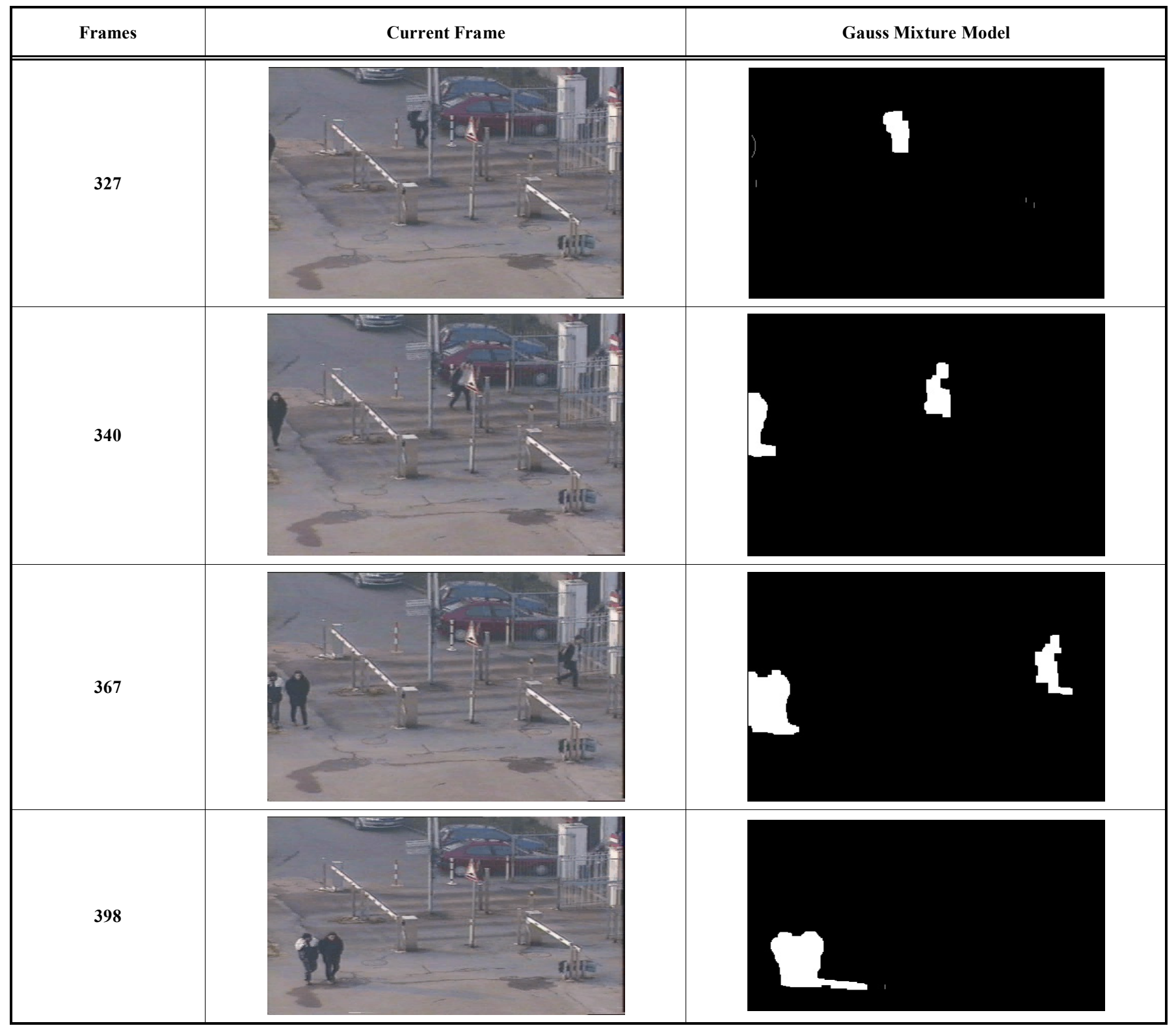

$\begin{cases}f_{t+1, i}(x, y)=f_{t, i}(x, y)+1 & l_{t, i}(x, y) \neq 255 \\ f_{t+1, i}(x, y)=f_{t, i}(x, y) & l_{t, i}(x, y)=255\end{cases}$

$l_{t+1, i}(x, y)=255$

For the unmatched model, the parameters are updated as below:

$$
\begin{aligned}
& \omega_{t+1, i}(x, y)=\omega_{t, i}(x, y) \\
& \mu_{t+1, i}(x, y)=\mu_{t, i}(x, y) \\
& f_{t+1, i}(x, y)=f_{t, i}(x, y) \\
& l_{t+1, i}(x, y)=l_{t, i}(x, y)-1
\end{aligned}
$$

There in, $\omega_{t, i}(x, y), \mu_{t, i}(x, y), f_{t, i}(x, y), l_{t, i}(x, y)$ are the weight, mean, frequency and survival time of the pixels $P(x, y)$ in the time and the ith model respectively. Sgn (number) is a symbol function (Table 2):

Table 2. Symbol function (1-2).

\begin{tabular}{|c|c|}
\hline Number & Sgn (number) \\
\hline \hline$>=0$ & 1 \\
\hline$=0$ & 0 \\
\hline$<=0$ & -1 \\
\hline
\end{tabular}


We devise a new method to update parameters when the initialization process of the model is completed. The model will quit when weight value $\omega$ and survival time $l$ of the model turn to be 0 and replace the original model with a new one. The parameter of the new model is set as the same as the video frame. The mean value of model parameter $\mu$ is the value of pixel, which means color or gray level, its weight $\omega$ is 1 , the survival time $l$ is set as 225 , and the frequency of occurrence $f$ is 0 .

Parameter update of the model matching and nonmatching is the same as formula 11 and 12 for not exiting model. The only difference is the way of updating weight.

If matching:

$\omega_{t+1, i}(x, y)=\omega_{t, i}(x, y)+N_{t}(x)-1$

If not matching:

$\omega_{t+1, i}(x, y)=\omega_{t, i}(x, y)-1$

There in $N_{t}(x)$ is the quantity of the model at pixel $P(x, y)$ in time t.

During building, simulation, updating, and exit of the model, the weight of pixel will increase or decrease depending on whether there exists a matching model. Weight will increase when the pixel matches some Gauss model, while weight will decrease when such a Gauss model does not exist. The distribution of weight will gradually focus on the background with large matching opportunity. The model mean value changes along with the variation of video frame pixel when matching, making the model adapt to the slow variation of the background. The survival time is $l$ when the model matches. And when the model mismatches, its survival time begins to count down and it won't time until the model completes its matching again. Reproducing frequency of the model is accumulative calculation frequency of model matching during the survival time of the model. The model will exit when the survival time of background model and recurrence frequency are zero [8-10]. Thus the background of short survival time and high frequency do not exit only because of reduction of weight reduction. For example, the small circulation changes exist in the background, such as the wind leaves, the flying flags, the rippling waves on water and so on. Therefore, this kind of design of algorithm, to a certain extent, possesses a stronger ability to tolerate a higher frequency noise than Guess model. Thus, it effectively suppresses noise and can further approach as well as simulate the background.

\section{THE EXPERIMENT AND ANALYSIS}

Firstly, we build the model for the background with our improved Gaussian mixture model. The process of initializing the video background is given in Table 3:

Exhibit in Table 3 also shows that background elements of the background image and the current frame are consistent when the $\mathrm{n}$ is 175 . Thus, the video monitoring system obtain a trusted background by improving Gaussian mixture model. Extraction of foreground in the background modeling by improved the Gauss mixture model and precision of new background difference module is guaranteed bases on the background.

Table 3. Background initialization (1-3).

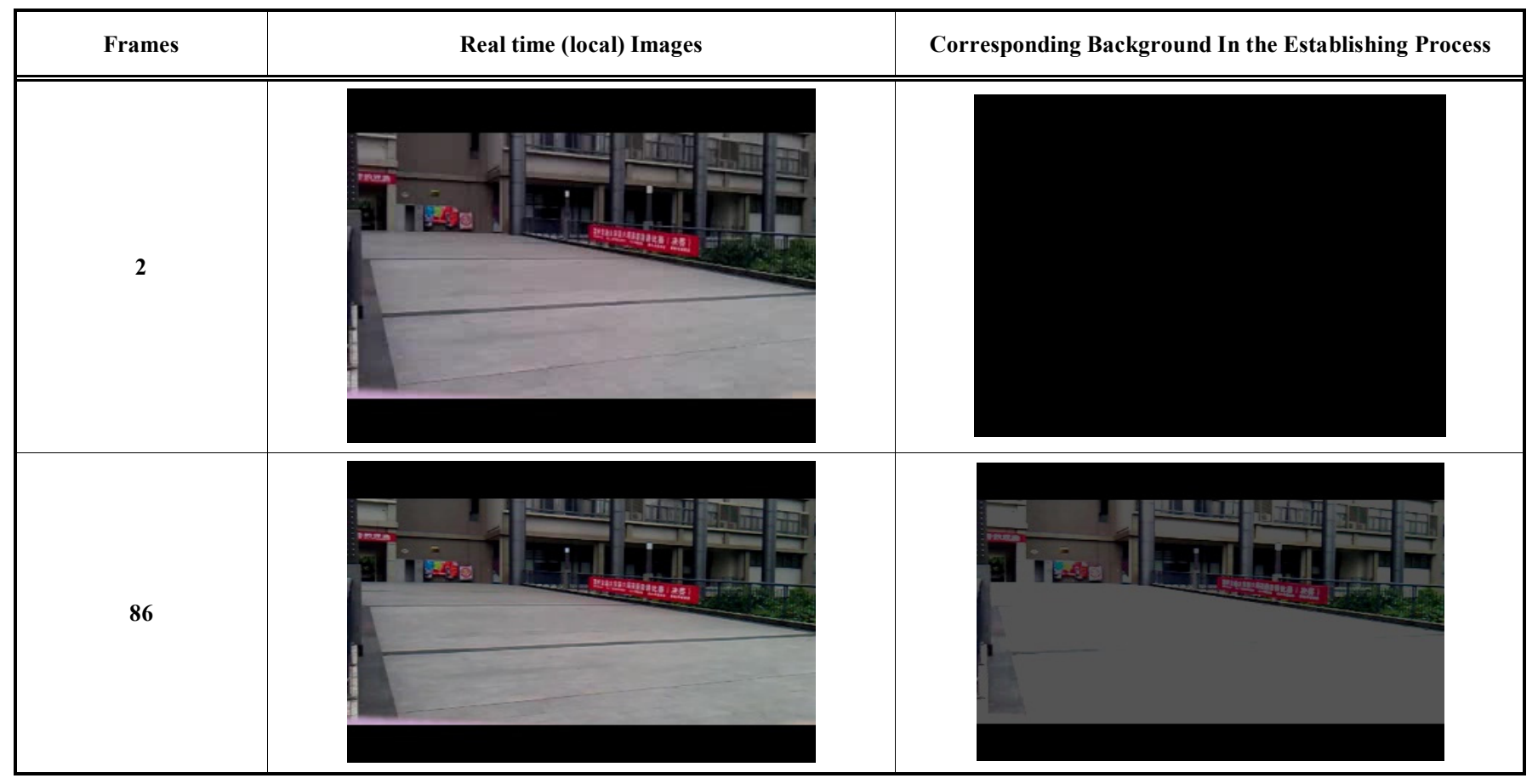

(Table 3) Contd.... 


\begin{tabular}{|l|c|c|c|}
\hline Frames & Real time (local) Images & Corresponding Background In the Establishing Process \\
\hline \hline 175 & & & \\
\hline
\end{tabular}

\subsection{The Method with an Improved Version on Gauss Mixture Model}

The performance with an improved Gauss mixture model building model is shown in Table $\mathbf{4}$ :

In terms of result contrast, though the extracted moving object is reliable, the result is still not so ideal. This is because the noisy components of the effective pixel are still existent in extraction results, of which the biggest is originated from the shadow of a moving object. The size of target shadow is uncertain since the position of light source is undetermined in simulation. The influence of the shadow is relatively insignificant when the light source is right above the target or the angle tilts slightly. However, the light source, such as that from the sun, will change from time to time. Or even a fixed light source is relative to the target location according to its movement. The size and length of the shadow is inversely proportional to the distance of the relative position.

The above experimental analysis implies that the operation of improved Gauss mixed background model has already provided a stable model for the system, while the key to the accuracy of the background difference depends on the precision and real-time performance of background image. Therefore, increasing the background difference module on the basis of mixed Gauss background model is feasible.

\subsection{The Method of Increasing Background Difference Module in Gauss Mixture}

When background difference increases, the real operation results of the experiment are shown in Table 5.

As is shown in Table 5, the inhibitory effect of this algorithm on the shadow is obvious. The result inherits the advantages of both Gaussian mixture background model and background difference. Our improved algorithm can effectively overcome the shadow and obviously inhibit any shadow of a moving target.

\subsection{The Contrast of the Algorithm and Background Difference Method}

If some of the video is obscure, choose a real surveillance video for contrast of the results of the algorithm and those of the background difference method:

The background difference method has an obvious inhibiting effect on the target shadow, and it can be analyzed from Table 6 that the effect of noise suppression of the algorithm adopted for this paper is far better than the background difference method, and the inhibiting effect of a target shadow is more ideal than the background difference method [10-13], giving users more convenience in the process of observation and analysis.

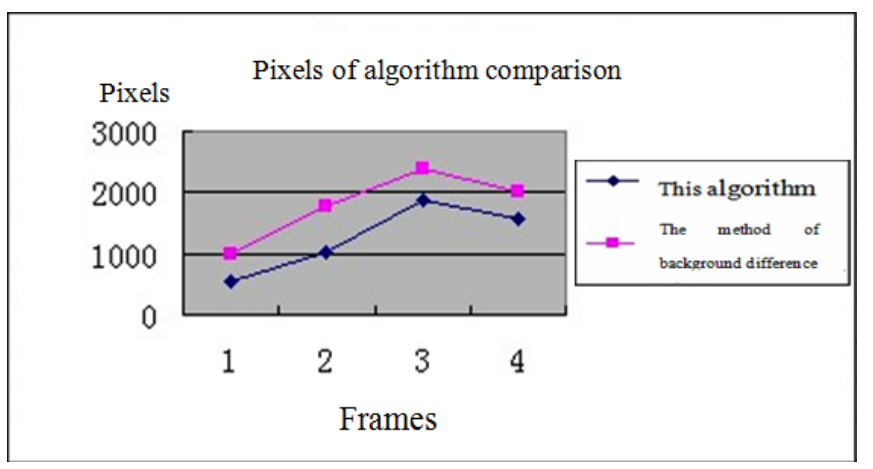

Fig. (1). Contrast of target pixels numbers under the real surveillance video.

It can be analyzed from (Fig. 1) that the pixel numbers of the algorithm are better than those of the background difference method. Under a high resolution, the advantages of this algorithm can be better reflected. 
Table 4. Performance of improved Gauss mixture model (1-4).

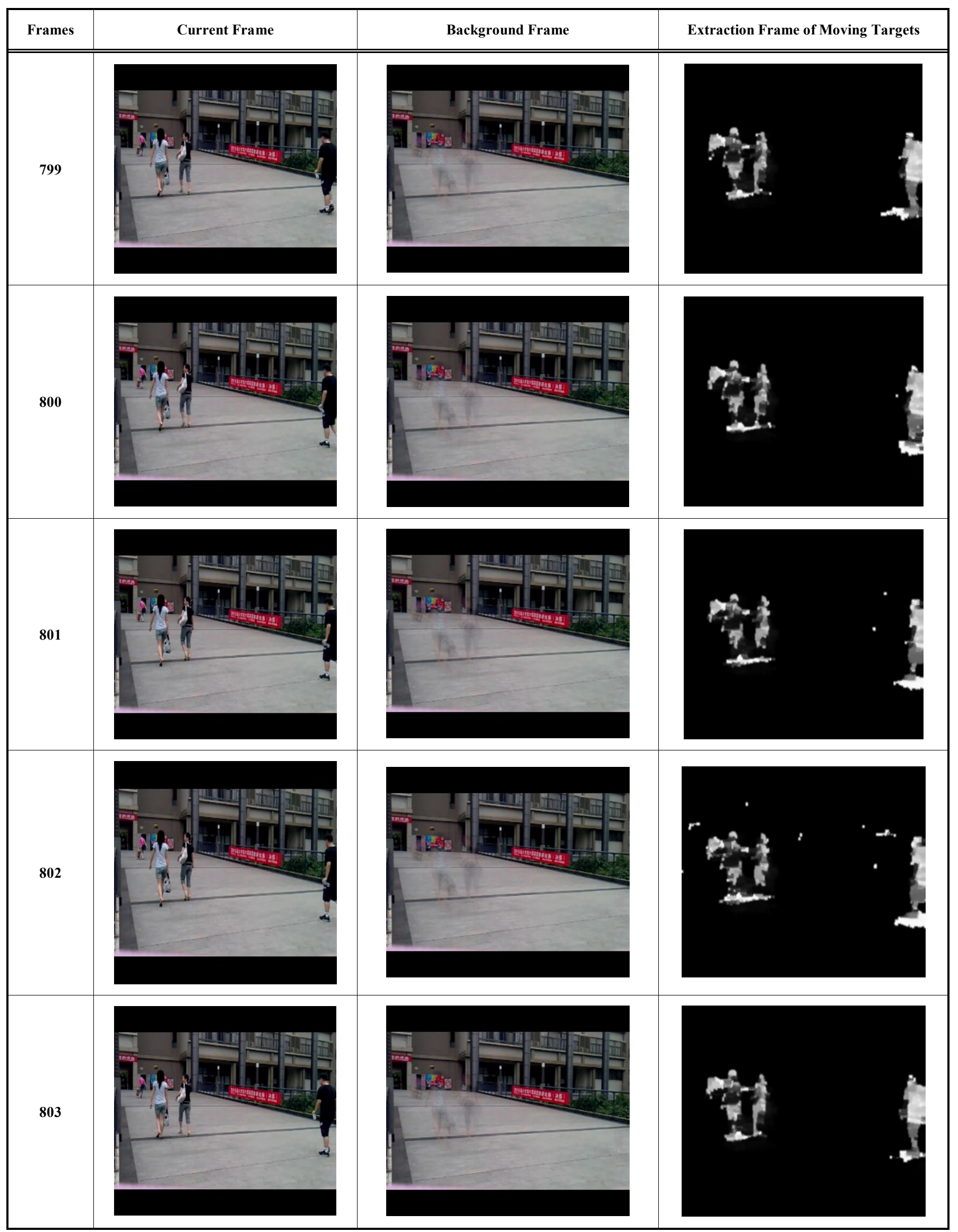


Table 5. Extraction of moving target with new algorithm (1-5).

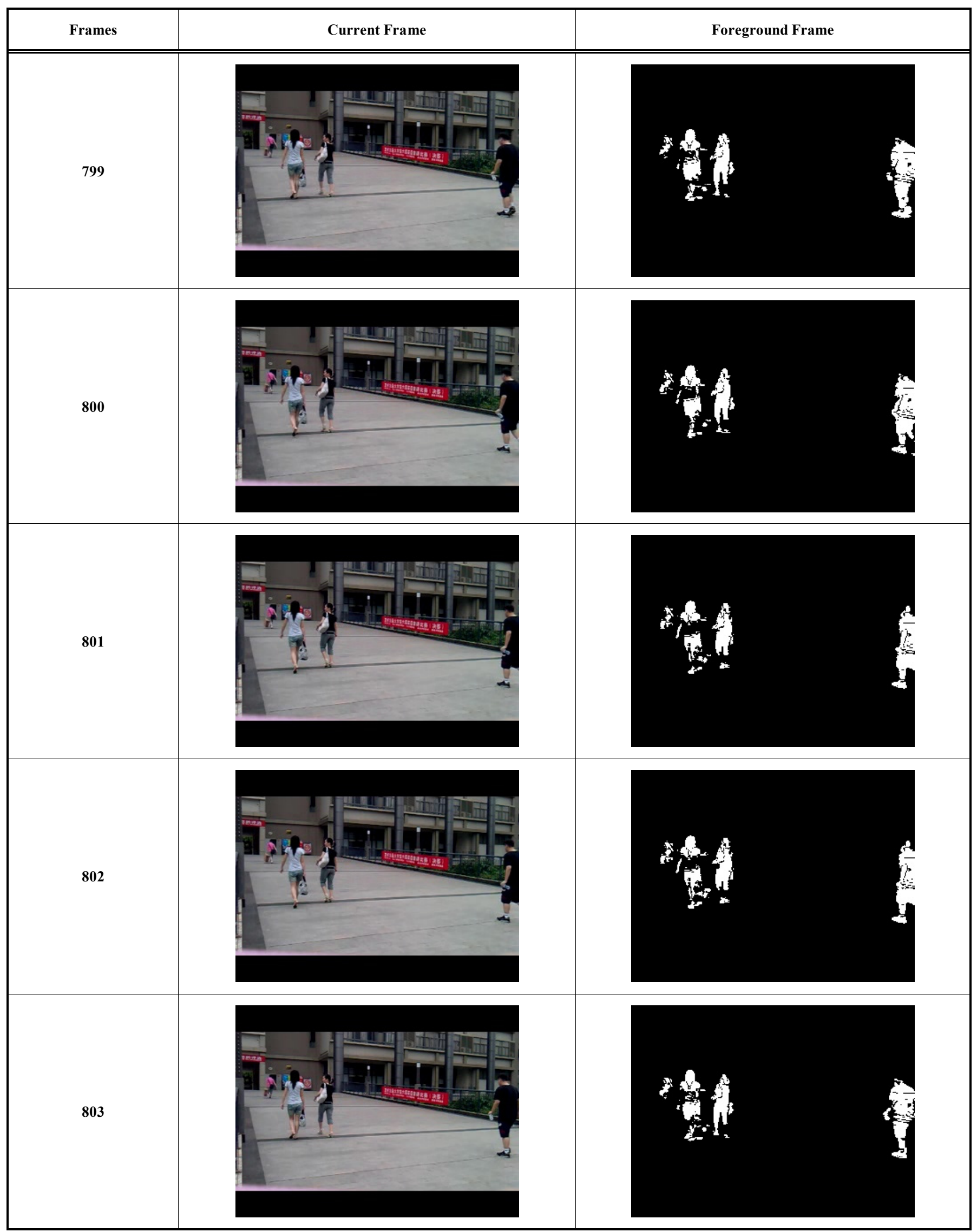


Table 6. Contrast of the algorithm and background difference method uuder the real surveillance video (1-6).

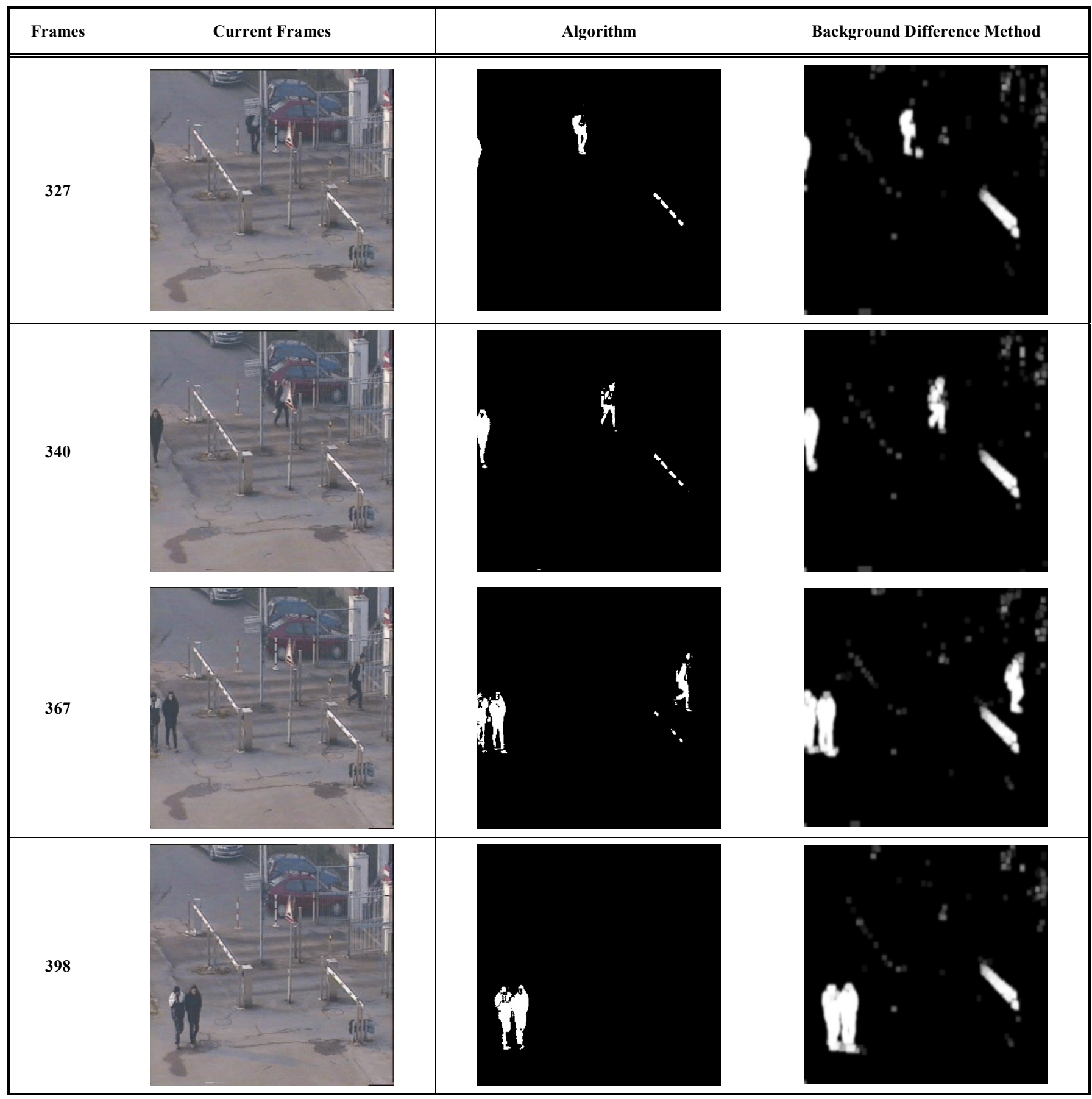

\subsection{The Contrast of Algorithm's Date with Classic} Mixture of Gaussian Background Model

In moving target detection, the modeling algorithm of classic Gaussian mixture background often occurs. In the following, we will compare with this paper's the modeling algorithm of the combination of background difference method and improved Gaussian mixture background.

In the comparison, the video is self recorded to simulate the working condition of outdoor environment's video input devices.
As shown in Table 7, the accuracy of the misdetection rate of two algorithms is not high. Besides, the classic Gaussian mixture background model on video processing is "not fluent", and more frequent.

As shown in Table 8, the speed of processing video of the modeling of the combination of background difference method and modified Gaussian mixture background is almost the same but faster than the modeling of classic Gaussian mixture background. 
Table 7. Miss rate (1-7).

\begin{tabular}{|c|c|c|c|}
\hline Modeling of classic Gaussian mixture background & 6930 & 204 & $2.943 \%$ \\
\hline
\end{tabular}

Table 8. Time of processing video (1-8).

\begin{tabular}{|c|c|c|}
\hline Name of Algorithm & Video Frame of Experiment & Rime of Processing Video \\
\hline \hline Modeling of classic Gaussian mixture background & 6930 & $00: 05: 28: 592$ \\
\hline Algorithm & 6930 & $00: 05: 26: 249$ \\
\hline
\end{tabular}

Table 9. Background rebuilt times (1-9) [14].

\begin{tabular}{|c|c|c|c|}
\hline Name of Algorithm & $\begin{array}{c}\text { Video Frame of } \\
\text { Experiment }\end{array}$ & $\begin{array}{c}\text { Background Change } \\
\text { Times }\end{array}$ & $\begin{array}{c}\text { Background Rebuilt } \\
\text { Times }\end{array}$ \\
\hline Bmodeling of classic Gaussian mixture background & 6930 & 8 & $8(100 \%)$ \\
\hline Balgorithm & 6930 & 8 & $3(37.5 \%)$ \\
\hline
\end{tabular}

As shown in Table 9, External factors as wind and pavement vibration (as shown in the experiment) have caused camera shake (a slide of the video equipment due to a larger shake), and the background has changed 8 times. In these changes, the classic Gaussian algorithm has rebuilt their background. This wide range simulation, directly leading to the detection of moving targets and disappearing in the window, affects the normal work of system. But the algorithms adopted by this paper simulated background only 3 times and on a small scale in the 8 background changes, and in all the 3 times simulations users' Observation and use are not so obviously affected. Although at some point in time or within a time slice, windows containing the amount of information are slightly more, the observation of moving targets is no problem [13]. This algorithm can be better adapted to the environment, which means it is highly robust.

\section{CONCLUSION}

In the detection of moving targets, it is suggested that the classical mixture Gaussian background modeling algorithm be used more. Although the Gaussian mixture model is partly able to eliminate the shadow, yet if the shadow area is larger, it will directly affect the extraction performance of moving objects. Through the processing of improvement, the Gaussian mixture modeling can obviously reduce the shadow area. But the algorithm of combined improved Gaussian mixture model is more advantageous than that of the background difference method in the present paper as it can completely eliminate the shadow. So the algorithm is better, and it has more advantages than Gaussian algorithm in terms of miss rate [15], processing time and numbers of background reconstruction. Therefore, it can better detect moving targets.

\section{CONFLICT OF INTEREST}

The author confirms that this article content has no conflict of interest.

\section{ACKNOWLEDGEMENTS}

The paper is founded by the National Natural Science Foundation Project (51208538), and the Open Fund Project of Ministry of Education (SLK2014B05).

\section{REFERENCES}

[1] L..Q. Shang-bing, and Linling, "Detection method of moving objects base on background difference and frame difference," Chinese Journal of scientific instrument, Shanghai, China, 2006.

[2] Z. Zivkovic, "Improved adaptive Gaussian mixture model for background substraction", In: Proceedings of the International Conference on Pattern Recognition, Amsterdam, Netherlands, 2004.

[3] P. KaewTraKulPong, and R. Bowden, An Improved Adaptive Background Mixture Model for Reahine Tracking with Shadow Detection, Kluwer Academic Publishers, Kingston, UK, 2001.

[4] D. S. Lee, "Improved adaptive mixture learning for robust video background modeling", In: Proceeding of IAPR Workshop on Machine Vision for Application, Nara, Japan, 2002.

[5] L. Liu, and Yun-hui, "Adaptive algorithm of multimodal fast background difference," China Journal of Image and Graphics, Henan, China, vol. 32, no. 5, pp. 452-455, 2008.

[6] Liujing, and Wangling, "An improved algorithm of Gaussian mixture model", Computer Engineering and Applications, Hebei, China, vol. 47, no. 18, pp. 624-627, 2010.

[7] C. Stauffer, and W. Crimson, "Learning patterns of activity using realtime tracking", IEEE Transactions on Pattern Analysis and Machine Intelligence, vol. 22, no. 8, pp. 747-757, Aug. 2000.

[8] R. Y. Mao, and X. N. Chen, "The improved HSI space morphology have noise of color image edge detection", Computer Application Research, vol. 42, no. 24, pp. 635-637, Feb. 2013.

[9] J. Liu, and L. Wang, "An improved algorithm of the gaussian mixture model Background method", Computer Engineering and Application, vol. 52, no. 30, pp. 168-170, 2010. 
[10] Y.Y. Wan, "Robustness of the morphology of color image edge detection algorithm", Computer Engineering and Application, vol. 54, no. 21, pp. 12-15, 2013.

[11] X. J. Huang, J.M. Zhou, and B.Y. Liu, "Adaptive mixture gaussian background model of moving target detection method," Computer Application, vol. 37, no. 24, pp. 835-839, 2010.

[12] L. Wang, and N.H.C. Yung, "Extraction of moving objects from their background based on multiple adaptive thresholds and boundary evaluation", Intelligent Transportation Systems, vol. 45, no. 20, pp. $40-51,2010$.
[13] G.Q. Luo, "Moving Target Detection and Tracking in Intelligent Video Surveillance," Master Thesis, Guangdong University of Technology, Guangdong of China, 2010.

[14] J.J. Duan, "Based on Color Space Power Battery Welding Point Positioning Technology Research", Master's Thesis, North central University, 2012.

[15] C. Ma, F. de Jun, and Z. Li, "Combined with threshold segmentation and morphological building shadow detection," Surveying and Mapping, vol. 26, no. 11, pp. 151-154, 2012.

(C) Liu Ying; Licensee Bentham Open.

This is an open access article licensed under the terms of the Creative Commons Attribution Non-Commercial License (http://creativecommons.org/licenses/by-nc/3.0/) which permits unrestricted, non-commercial use, distribution and reproduction in any medium, provided the work is properly cited. 